\title{
Numerical Tokamak Project Code Comparison
}

\author{
R.E. Waltz \\ B.I. Cohen \\ M.A. Beer \\ J.A. Byers \\ A.M. Dimits \\ W. Dorland \\ S.E. Parker \\ R.A. Santoro \\ R.D. Sydora \\ H.V. Wong
}

June 21, 1994

This is an informal report intended primarily for internal or limited external distribution. The opinions and conclusions stated are those of the author and may or may not be those of the Laboratory.

Work performed under the auspices of the U.S. Department of Energy by the Lawrence Livermore National Laboratory under Contract W-7405-Eng-48. 


\section{DISCLAIMER}

This document was prepared as an acccount of work sponsored by an agency of the United States Government. Neither the United States Government nor the University of California nor any of their employees, makes any warranty, express or implied, or assumes any legal liability or responsibility for the accuracy, completeness, or usefulness of any information, apparatus, product, or process disclosed, or represents that its use would not infringe privately own rights. Reference herein to any specific commercial products, process, or service by trade name, trademark, manufacturer, or otherwise, does not necessarily constitute or imply its endorsement, recommendation, or favoring by the United States Government or the University of California. The views and opinions of authors expressed herein do not necessarily state or reflect those of the United States Government or the University of California, and shall not be used for advertising or product endorsement purposes.

This report has been reproduced directly from the best available copy.

Available to DOE and DOE contractors from the Office of Scientific and Technical Information P.O. Box 62, Oak Ridge, TN 37831

Prices available from (615) 576-8401, FTS 626-8401

Available to the public from the National Technical Information Service

U.S. Department of Comunerce 5285 Port Royal Rd. Springfield, VA 22161 
DISCLAIMER

Portions of this document may be illegible in electronic image products. Images are produced from the best available original document. 


\title{
Numerical Tokamak Project Code Comparison
}

\author{
R.E. Waltz, ${ }^{1}$ B. I. Cohen, ${ }^{2}$ M.A. Beer, ${ }^{3}$ J.A. Byers, ${ }^{2}$ A. M. Dimits, ${ }^{2}$ \\ W. Dorland, ${ }^{3}$ S.E. Parker, ${ }^{3}$ R.A. Santoro, ${ }^{3}$ \\ R. D. Sydora, ${ }^{4}$ and H. V. Wong 5 \\ ${ }^{1}$ General Atomics, San Diego, CA 92186 \\ 2Lawrence Livermore National Laboratory, Livermore, CA 94550 \\ 3Princeton Plasma Physics Laboratory, Princeton, NJ 08540 \\ ${ }^{4}$ University of California at Los Angeles, Los Angeles, CA 90024 \\ 5University of Texas at Austin, Austin, TX 78712
}

\begin{abstract}
The Numerical Tokamak Project undertook a code comparison using a set of TFTR tokamak parameters. Local radial annulus codes of both gyrokinetic and gyrofluid types were compared for both slab and toroidal case limits assuming ion temperature gradient mode turbulence in a pure plasma with adiabatic electrons. The heat diffusivities were found to be in good internal agreement within $\pm 50 \%$ of the group average over five codes.
\end{abstract}


In March 1994, the Numerical Tokamak Project (NTP) completed a code comparison using a set of standard case parameters from TFTR suggested by G. Hammett and M. Zarnstorff (PPPL). In 1992-93 many groups participating in the NTP were doing electrostatic ion temperature gradient mode turbulence simulations with adiabatic electrons and pure ion plasmas. While this does not encompass many aspects of turbulence in tokamaks, it is believed to be a good initial paradigm with easily standardized physics. This made the problem well suited to a code comparison.

The compared codes are of three types: gyro-landau fluid (GLF) presented by Beer, Dorland, and Waltz; gyrokinetic particle (GKP) presented by Byers, Dimits, Sydora, and Santoro; and gyrokinetic fluid (GKF) presented by Wong. The details for most of these codes may be found in the references below. Two types of simulations were compared: "slab" and "toroidal", the former with curvature and $\nabla \mathrm{B}$ drifts and the magnetic mirroring force excluded. These particular codes also have the important common feature that they are all local homogeneous turbulence models and represent the turbulence in a narrow radial annulus with cyclic boundary conditions: eddies leaving one side cyclically enter the other. This is believed at present to be a reasonable first approximation if the eddies and radial correlation lengths are small compared to the radial width of the box and the temperature gradient lengths. As long as the simulations are insensitive to the numerical grid density, the transport must then scale to the gyro-Bohm unit $c_{s} \rho_{s}^{2} / L_{T}$ where $c_{S}=\left(T / m_{i}\right)^{1 / 2}$ is the sound speed and $\rho_{\mathrm{S}}=\mathrm{c}_{\mathrm{S}} / \Omega$ is the ion gyroradius $\left(\mathrm{T}_{\mathrm{e}}=\mathrm{T}_{\mathfrak{j}}\right)$ with the ion cyclotron frequency $\Omega=e B / m_{i} c$ and $L_{T}$ the temperature gradient length. Furthermore, the cross-field wavelengths scale to $\rho_{\mathrm{s}}$, and the growth rates and frequencies scale to $\mathrm{c}_{\mathrm{S}} / \mathrm{L}_{\mathrm{T}}$. Thus we have a convenient system of units. This annulus approach has the added virtue that it is easy to hold the temperature gradients fixed and avoid any quasilinear relaxation of the gradients driving the turbulence. These are all features which allow easy standardization of the problem. We expect that future work with global codes, which allow turbulent energy to leave local annuli and the eddy sizes to be influenced by variation in the plasma conditions across the radius, will be required to determine the scaling with $\rho_{\mathrm{S}} / L_{\mathrm{T}}$. All the codes presented here are believed to have gyro-Bohm scaling.

The inclusion of "radial" modes was found to be an important physics consideration required to get meaningful comparison. These modes have toroidal mode number $\mathrm{n}=0$ (and $\mathrm{m}=0, \pm 1$ ). In a slab model the "radial" modes correspond to those modes with $\mathrm{k}_{\mathrm{z}}=\mathrm{k}_{\mathrm{y}}=0$ and $\mathbf{k}_{\mathrm{x}} \neq 0$. They correspond to nonlinearly driven $\mathbf{E} \times \mathbf{B}$ rotational shears that have an important stabilizing effect on the turbulence. (They also provide local steepening and flattening of the driving temperature gradient.). The physics of these modes appears to be 
crucial and the adiabatic electron assumption must be carefully specified by $\tilde{\mathrm{n}} / \mathrm{n} 0=\left(\mathrm{e} / \mathrm{T}_{\mathrm{e}}\right)(\phi-$ $\langle\phi\rangle$ ) where $\langle\phi\rangle$ is a field-line-averaged potential. All the simulations presented made these common assumptions.

The parameters of the code comparison are from a deuterium TFTR L-mode (Shot No. 41309) [S.D. Scott, et al, Phys. Fluids B 2, 1300 (1990)] at mid radius $r / a=0.576$. The relevant parameters for the simulations are $\eta_{\mathrm{i}}=\mathrm{L}_{\mathrm{n}} / \mathrm{L}_{\mathrm{T}}=4, \mathrm{~T}_{\mathrm{j}} / \mathrm{T}_{\mathrm{e}}=1, \mathrm{q}=2.4, \mathrm{~s}=$ $(\mathrm{d} \ln \mathrm{q} / \mathrm{d} \ln \mathrm{r})=1.5, \mathrm{R} / \mathrm{L}_{\mathrm{T}}=10$. For the slab simulations, only the combination $\mathrm{L}_{\mathrm{T}} / \mathrm{L}_{\mathrm{S}}=0.0625$ where $\mathrm{L}_{\mathrm{S}}=\mathrm{Rq} / \mathrm{s}$ enters. Other parameters of interest are $\rho_{\mathrm{S}} / \mathrm{L}_{\mathrm{T}}=0.0056$, $\mathrm{R} / \mathrm{a}=2.8, \mathrm{a}=0.92 \mathrm{~m}, \mathrm{~B}=3.8 \mathrm{~T}, \mathrm{n}=3.3 \times 10^{19} \mathrm{~m}^{-3}, \mathrm{Z}_{\mathrm{eff}}=2.2$. A TFTR supershot (Shot No. 44669) has previously been the focus of detailed analysis [W. Horton, D. Lindberg, et al., Phys. Fluids B 4, 953 (1992)].

S The experimental effective heat diffusivity was $2.2 \mathrm{c}_{\mathrm{s}} \rho_{\mathrm{s}}^{2} / \mathrm{L}_{\mathrm{T}}$. Again we caution that the codes are considering the idealized case of a $\beta=0$ pure ion plasma with adiabatic electrons. Impurity gradient effects are known to have important stabilizing effects, and trapped electrons are known to have important destabilizing effects under these particular plasma conditions. Thus, any close proximity to this value of heat diffusivity from the codes may be fortuitous.

The slab simulations are presented in Table 1 and the toroidal simulations in Table 2. Figures 1 and 2 present the corresponding results for the heat diffusivity in graphical form. In the slab case, the diffusivity is within $\pm 50 \%$ of the average $(0.0275)$ (excluding Byers' result) and in the toroidal case also within $\pm 50 \%$ of the average $(0.817)$. Considering the deviations from different mesh sizes, the fluctuations in the time histories, the statistical noise in the GKP simulations, and the fact that scaling is of more interest than exact magnitude, we regard this as good agreement! Note that the toroidal results are consistently 20-30 fold larger than slab results because of the important curvature and $\nabla \mathrm{B}$ destabilization. The toroidal results are comparable to experimental diffusion levels as we would hope.

Slab case comparisons among codes at PPPL are discussed in detail in Parker et. al. [Phys. Plasmas 1, 1461 (1994)]. Discussing the toroidal case in somewhat more detail here, the growth rates of the fastest mode are within $\pm 30 \%$ of the average $(0.0375)$. The result from an accurate gyrokinetic stability code GKS [M. Kotschenreuther, Bull. Am. Soc 37, 1432 (1991)] is 0.0336 . The deviation in the diffusivity does not necessarily follow the deviation in the growth rate. In some cases one model is known to be better than another. For example, Beer used a 6-moment GLF model which gives excellent agreement with the GKS code, whereas Waltz used a simple 4-moment model which is known to give lower growth rates. There is also some dependence of the results on spatial resolution 
characteristics: for example, Waltz obtained $28 \%$ higher diffusion with a $3 \times$ more dense wavenumber mesh. Differences in diffusion, however, were not always accounted for by mesh, model, or technique. For example, Beer and Waltz made a separate comparison with an identical 4-moment GLF model with the same spectral resolution, obtaining exactly the same linear spectrum (with $1 \%$ differences in growth rates), but Beer's result was about $30 \%$ systematically lower than Waltz's result for the diffusivity.

For completeness, Table 3 contains some of the computational details on grid sizes, particle numbers, timings, etc. All cases were run on the C-90 at NERSC. Several of the codes have been run on the CM-200 and CM-5 at the Advanced Computing Laboratory at Los Alamos National Laboratory. In the process of doing the code comparisons, it was discovered that using the same spatial filtering algorithm was important in bringing the results into closer quantitative agreement. The code comparison activity was also instructive in determining convergence characteristics with respect to mesh resolution and particle statistics. One should note that in Table 3 the GLF simulations of Beer and Waltz use a significantly smaller number of modes than do the GKP simulations of Parker and Dimits. This apparent difference is deceptive, however. The GKP simulation model uses a spatial grid on which charge densities are obtained by interpolation from the particle positions and from which forces are interpolated back to the particle positions to accelerate the particles, while the GLF model uses a spectral model. Because of the grid, short wavelength modes in the GKP model are distorted. The grid-induced distortion scales as $(k \Delta x / 2)^{2}$, and roughly half of the Fourier modes in each spatial dimension are significantly distorted. The use of spatial filtering also limits the amount of the wavelength spectrum that is undistorted. Thus, the number of weakly distorted mode pairs in Table 3 for the GKP simulation models is less than $1 / 4$ of the number of pairs given there; and the spatial resolutions of the GKP and GLF simulations are comparable.

\section{Acknowledgment}

Research on the Numerical Tokamak Project is supported jointly by the Office of Fusion Energy and the Office of Scientific Computing as part of the High Performance Computing and Communications Initiative through contracts and grants from the U.S. Department of Energy to the participating institutions. Computing resources and assistance was provided by the National Energy Research Supercomputer Center at Lawrence Livermore National Laboratory and the Advanced Computing Laboratory at Los Alamos National Laboratory. 
Table 1. Slab Simulations

Research Group Dorland Waltz Byers Sydora Santoro Parker

code type

GLF GLF GKP GKP GKP GKP

$\chi \mathrm{L}_{\mathrm{T}} /\left(\mathrm{c}_{\mathrm{s}} \rho_{\mathrm{s}}^{2}\right)$

$0.033 \quad 0.040$

0.11

0.015

0.02

0.03

average

$\pm 0.005$

$\chi \mathrm{L}_{\mathrm{T}} /\left(\mathrm{c}_{\mathrm{s}} \rho_{\mathrm{s}}{ }^{2}\right)$

0.044

$0.02-0.08$

0.11

0.03-0.05

1 st time peak

$(e \phi / T) L_{T} / \rho_{s}$

0.25

0.55

1.4-0.3

0.18

0.01

0.25

rms

$\mathrm{k}_{\theta} \rho_{\mathrm{s}}$

0.3

0.5

0.25

0.4

0.2

spec. peak

$\gamma \mathrm{L}_{\mathrm{T}} / \mathrm{c}_{\mathrm{s}}$

0.015

0.012

0.011

0.02

0.015

0.019

fastest mode

$\omega \mathrm{L}_{\mathrm{T}} / \mathrm{c}_{\mathrm{s}}$

$-0.042$

$-0.048$

$-0.052$

$-0.05$

$-0.051$

$-0.05$

fastest mode

$\mathrm{k}_{\theta} \rho_{\mathrm{s}}$

0.5

0.5

0.5

0.5

0.5

0.5

fastest mode

$\left(\Delta x / \rho_{\mathrm{s}}\right)_{\mathrm{rms}}$

1.25

0.93

$1.5-2$

2

2

fastest mode

$\eta_{\text {i_crit }}$

-2.5
$-\quad$.

1.6

2.1

1.5 
Table 2. Toroidal Simulations

Research Group Beer Waltz Parker Wong Dimits

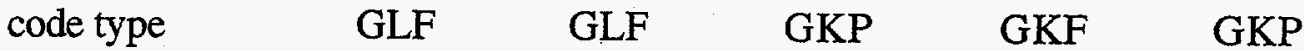

$\begin{array}{llllll}\chi \mathrm{L}_{\mathrm{T} /\left(\mathrm{c}_{\mathrm{s}} \rho_{\mathrm{s}}{ }^{2}\right)} & 1.2 & 0.553 & 0.73 & 1.0 & 0.6\end{array}$

average

$\chi \mathrm{L}_{\mathrm{T}} /\left(\mathrm{c}_{\mathrm{s}} \rho_{\mathrm{s}}{ }^{2}\right)$

$\begin{array}{llll}- & 0.27-1.0 \quad \ldots \quad & \ldots\end{array}$

1st time peak

$\begin{array}{lllll}(\mathrm{e} \phi / \mathrm{T}) \mathrm{L}_{\mathrm{T}} / \rho_{\mathrm{S}} & 2.5 & 1.8 & 4 & 2.2\end{array}$

rms_flux avg

$\begin{array}{llllll}\mathrm{k}_{\theta} \rho_{\mathrm{s}} & 0.15 & 0.2 & 0.1 & - & 0.1\end{array}$

spec. peak

Flow asymmetry $\quad$ - $\quad 10: 1$

out : in

Turbulence $\quad 2: 1 \quad 3: 2$

out $:$ in

$\begin{array}{llllll}\gamma \mathrm{L}_{\mathrm{T}} / \mathrm{c}_{\mathrm{s}} & 0.035 & 0.023 & 0.047 & 0.04 & .045\end{array}$

fastest mode

$\begin{array}{llllll}\omega \mathrm{LT}_{\mathrm{T}} / \mathrm{c}_{\mathrm{S}} & -0.16 & -0.136 & -0.18 & -0.15 & -.085--.13\end{array}$

fastest mode

$\begin{array}{llllll}\mathrm{k}_{\theta} \rho_{\mathrm{S}} & 0.45 & 0.4 & 0.49 & 0.45 & 0.37-0.49\end{array}$

fastest mode

$\eta_{i \_c r i t}$

2.7

2.9

$-\quad<2.5$ 
Slab Simulation

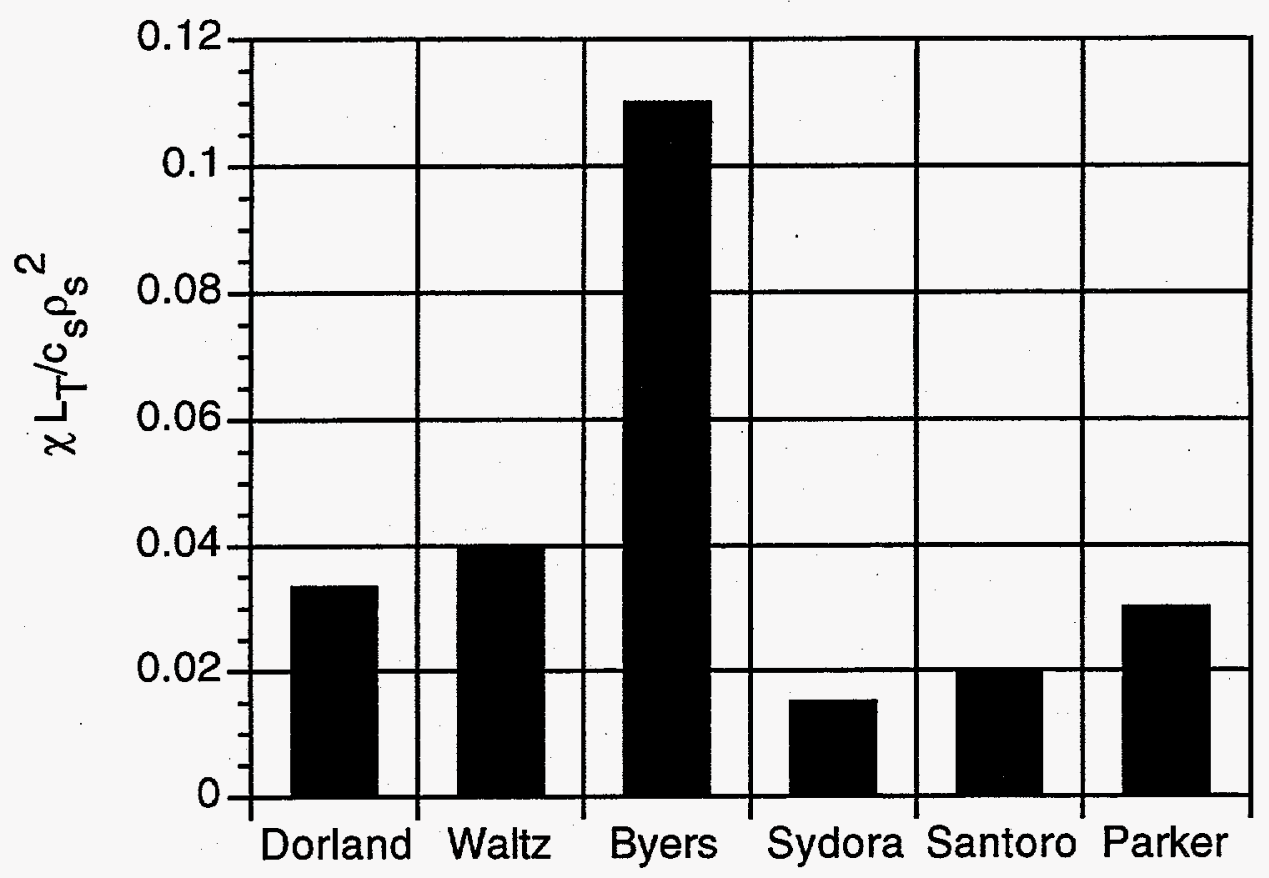

Figure 1. Slab simulations. Excluding Byers all results with $\pm 50 \%$ of group average value 0.0275 . 
Toroidal Simulation

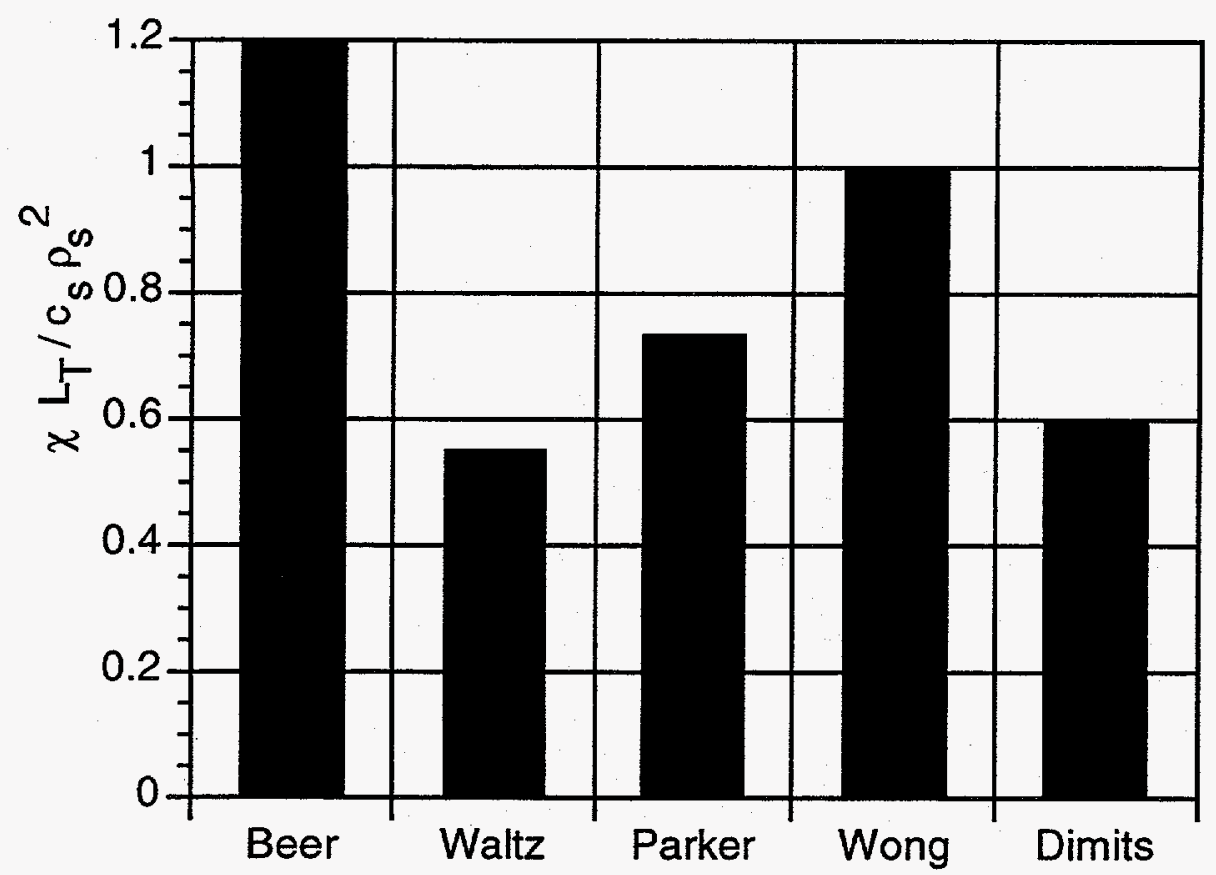

Figure 2. Toroidal Simulation. All within $\pm 50 \%$ of group average value 0.817 . 
Table 3. Computational Parameters

\begin{tabular}{|c|c|c|c|c|c|}
\hline Research Group & Beer & Waltz & Parker & Wong & Dimits \\
\hline $\begin{array}{l}\text { time step }{ }^{\times} \mathrm{c}_{\mathrm{S}} / \mathrm{L}_{\mathrm{T}} \\
\text { nonlinear phase }\end{array}$ & 0.04 & 0.18 & 0.1 & 0.064 & 0.2 \\
\hline number of steps & 16000 & 15000 & 2000 & - & 4000 \\
\hline CPU/step (sec) & 2 & 1 & 4.2 (1 proc.) & - & 6-12 (1 proc.) \\
\hline total CPU (hrs) & 12 & 4 & 2.8 (1 proc.) & - & 7 \\
\hline wall clock & $3-4$ & 6 & 0.2 & - & $0.4-0.8$ \\
\hline turn around & $4 \mathrm{cpu}$ & $1.5 \mathrm{cpu}$ & $(14 \mathrm{cpu})$ & & (12 cpu) \\
\hline code size (MW) & 27 & 40 & 20 & & $54-107$ \\
\hline radial grid $=j$ & 96 & 64 & 64 & - & $64-128$ \\
\hline $\begin{array}{l}\text { no. }(\mathrm{m} . \mathrm{n}) \text { or } \\
(\mathrm{ky}, \mathrm{kz}) \text { pairs=k }\end{array}$ & 1318 & 850 & 4096 & - & $2000-8000$ \\
\hline no. particles $=p$ & 0 & 0 & $0.5 \mathrm{M}$ & - & $2 \mathrm{M}-4 \mathrm{M}$ \\
\hline size and time $=$ & & & & & \\
\hline$j \times k+p$ & $\mathrm{j} \times \mathrm{k}$ & $j \times k$ & $\mathrm{j} \times \mathrm{k}+\mathrm{p}$ & - & $\mathrm{j} \times \mathrm{k}+\mathrm{p}$ \\
\hline $\mathrm{k}_{\theta} \rho_{\mathrm{S} \_} \max$ & 0.951 & 1.0 & - & - & 3 \\
\hline $\mathrm{k}_{\theta} \rho_{\mathrm{S} \_} \_$min & 0.063 & 0.1 & - & - & 0.125 \\
\hline
\end{tabular}




\section{References}

Beer

M. A. Beer, S. C. Cowley, and G. W. Hammett, "Field-aligned Coordinates for Nonlinear Simulations of Tokamak Turbulence," Princeton University (May 26, 1994), to be submitted to Physics of Plasmas.

G. W. Hammett, M. A. Beer, W. Dorland, S. C. Cowley, and S. A. Smith, "Developments in Gyrofluid Approach to Tokamak Turbulence Simulations", Plasma Phys. Controlled Fusion 35, 973 (1993).

Byers

B. I. Cohen, T. J. Williams, A. M. Dimits, and J. A. Byers, "Gyrokinetic Simulations of $\mathbf{E} \times \mathbf{B}$ Velocity-Shear Effects on Ion-Temperature-Gradient Modes," Phys. Fluids B 5, 2967 (1993).

Dorland

William Dorland, "Gyrofluid Models of Plasma Turbulence," $\mathrm{PhD}$ Thesis, Princeton University, 1993.

W. Dorland and G.W. Hammett, "Gyrofluid Turbulence Models with Kinetic Effects," Phys. Fluids B 5, 812 (1993) .

Dimits

A.M. Dimits, "Fluid Simulations of Tokamak Turbulence in Quasiballooning Coordinates," Phys. Rev. E 48, 4070 (1993).

A.M. Dimits and B.I. Cohen, "Simulation Models for Tokamak Plasmas,"in Proc. of the 14th IAEA Conference on Advances in Simulation and Modeling of Thermonuclear Plasmas, IAEA Technical Meeting, Montreal,15-17 June 1992 (Vienna, 1993). 
Parker

S.E. Parker, W. Dorland, R.A. Santoro, M.A. Beer, Q.P. Liu, W.W. Leee, G.W. Hammett,"Comparisons of Gyrofluid and Gyrokinetic Simulation," Phys.

Plasmas 1, 1461 (1994)

S.E. Parker, W.W. Lee, and R.A. Santoro, "Gyrokinetic Simulation of Ion Temperature Gradient Driven Turbulence in 3D Toroidal Geometry," Phys. Rev. Lett. 71, 2042 (1993).

Santoro

R.A. Santoro, Ph.D. Thesis, Princeton University (1993)

Sydora

R. D. Sydora, "3D Gyrokinetic Particle Simulations of Ion Temperature GradientDriven Turbulent Transport", Proceedings of the 1993 US-Japan Workshop on Ion Temperature Gradient Driven Turbulent Transport, Ed. W. Horton, M. Wakatani, and A. Wooten, American Institute of Physics, 1993.

R. D. Sydora, T.S. Hahm, W.W. Lee, and J.M. Dawson, "Dynamics and Fluctuation Spectrum of Ion Temperature Gradient Drift Instabilities", Phys. Rev. Lett. 64, 2015, 1990.

Waltz

R.E. Waltz, G.D Kerbel, and J. Milovich,"Toroidal gyro Landau fluid model turbulence simulations with a nonlinear ballooning mode representation", General Atomics Report GA-A21565 to be published in Physics of Plasmas, July 1994

Wong

unpublished 